
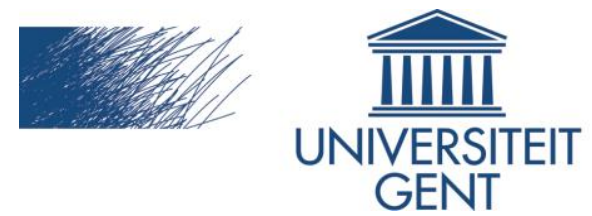

biblio.ugent.be

The UGent Institutional Repository is the electronic archiving and dissemination platform for all UGent research publications. Ghent University has implemented a mandate stipulating that all academic publications of UGent researchers should be deposited and archived in this repository. Except for items where current copyright restrictions apply, these papers are available in Open Access.

This item is the archived peer-reviewed author-version of: Efficient Innate Immune Killing of Cancer Cells Triggered by Cell-Surface Anchoring of Multivalent Antibody-Recruiting Polymers

Authors: Uvyn A., De Coen R., Gruis M., Tuk C.W., De Vrieze J., van Egmond M., De Geest B. In: Angewandte Chemie-International Edition 58(37): 12988-12993

To refer to or to cite this work, please use the citation to the published version:

Uvyn A., De Coen R., Gruis M., Tuk C.W., De Vrieze J., van Egmond M., De Geest B. (2019) Efficient Innate Immune Killing of Cancer Cells Triggered by Cell-Surface Anchoring of Multivalent Antibody-Recruiting Angewandte Chemie-International Edition 58(37): 12988-12993

DOI: 10.1002/anie.201905093 


\title{
Efficient innate immune killing of cancer cells triggered by cell surface anchoring of multivalent antibody-recruiting polymers
}

\author{
Annemiek Uvyn, ${ }^{\#[a]}$ Ruben De Coen, ${ }^{[a]}$ Mandy Gruijs, ${ }^{[b]}$ Cees W. Tuk, ${ }^{[b]}$ Jana De Vrieze, ${ }^{[a]}$ \\ Marjolein van Egmond, ${ }^{[b]}$ Bruno G. De Geest*[a]
}

[a] Annemiek Uvyn, Dr. Ruben De Coen, Jana De Vrieze,Prof.Dr. Bruno G. De Geest

Department of Pharmaceutics, Ghent University, Belgium

[b] Mandy Gruijs, Cees W. Tuk, Prof.Dr. Marjolein van Egmond

Department of Molecular Cell Biology and Immunology, Amsterdam UMC, location VUmc, Amsterdam, the Netherlands.

\# These authors contributed equally to the work.

\begin{abstract}
Rewiring innate immune defence to eradicate cancer is one of the mechanisms of action of several monoclonal antibodies (mAbs) used in the clinic. Binding of mAbs onto a cell surface triggers antibodymediated effector killing by innate immune cells and through complement activation. As an alternative to mAbs, which face inherent issues with costs of production and stability, synthetic systems that can recruit endogenous antibodies from the blood stream to a cancer cell surface could be of great relevance. Here we explore antibody-recruiting polymers (ARPs) as a novel class of immunotherapy. ARPs consist of a cell binding motif linked to a polymer that contains multiple small molecule antibody-binding motifs along its backbone. As a proof of concept, we employ a lipid anchor that inserts into the phospholipid cell membrane and make use of a polymeric activated ester scaffold onto which we substitute dinitrophenol as antibody-binding motif. We demonstrate that ARPs allow for high avidity antibody binding and are able to drive antibody recruitment to treated cells for up to several days. Further we show that ARP-treated cancer cells are prone to antibody-mediated killing of cancer cells through phagocytosis by macrophages.
\end{abstract}

\section{Manuscript}

Exploiting innate immune effector mechanisms against the Fc domain of monoclonal antibodies (Abs) is gaining immense popularity in anti-cancer therapy and also holds promise for the treatment of infectious diseases. ${ }^{[1][2]}$ Its mechanism of action relies on clustering of Abs onto a target (cancer) cell surface by binding with its Fab fragments to a protein expressed on the target cell surface. Subsequent exposure of the $\mathrm{Fc}$ domain ${ }^{[3]}$ towards the external medium flags the cell for destruction by a combination of complement activation, NK-cell mediated killing and phagocytosis by macrophages. ${ }^{[4-6]}$ The latter two mechanisms directly depend on recognition of Fc domains on the target cell surface by Fc receptors on the innate effector cells. ${ }^{[3]}$

As an alternative to the use of externally administered monoclonal Abs, exploiting the presence of endogenous antibodies that are present in the serum of every human being, ${ }^{[7]}$ could be beneficial as it circumvents the need for recombinant production and genetic engineering to make the Ab compatible with the host as well as avoiding stability issues often encountered with long term storage of mAbs. In the context of using endogenous Abs, the main questions are (1) which Abs to exploit and (2) how to 
recruit these to a target cell surface. The first question has been addressed by several groups and mainly focuses on the presence of antibodies in human blood against galactose- $\alpha-1,3$-galactose, phosphorylcholine, rhamnose or dinitrophenol (DNP). ${ }^{[8]}$ The nature of these endogenous antibodies varies from the consumption of meat (galactose- $\alpha-1,3$-galactose), commensal bacteria in the gut (phosphorylcholine and rhamnose) and exposure to pesticides (DNP). The second question relates to antibody recruitment, which involves the process that drives binding of endogenous antibodies to a target cell surface. In this regard, hydrophobic interaction, which typically relies on a lipid motif conjugated to an antibody-binding motif, has been explored. ${ }^{[9]}$ The lipid part anchors the cell membrane and exposes the antibody-binding motif to the external medium. This approach is intended for local application, i.e. by direct intratumoral injection, due to the lack in selectivity towards cancer cells. Another strategy involves conjugates composed of an antibody-binding motif and a target cell binding motif. For the latter, several cell surface receptors that are overexpressed by cancer cells have been explored, including the folate receptor, ${ }^{[10]}$ PSMA, ${ }^{[11]}$ UPAR, ${ }^{[12]}$ and others. These approaches all involve mono- or at best bi-valent molecules, both on the level of the antibody-binding as well as the target cell binding motifs.

The question we aim to address in this paper is whether polymeric scaffolds that contain multiple motifs of an antibody-recruiting motif allow for a more efficient antibody recruitment to the cell surface with prolonged cell surface persistence and efficient induction of innate effector killing. Hereto we explore the concept of so-called antibody-recruiting polymers (ARPs). These consist of multiple copies of on the one hand motifs that bind to endogenous antibodies and on the other hand a motif that anchors to the cell surface, thereby tagging the cell for attack by the innate immune system (Scheme 1).

\section{antibody recruiting polymer}

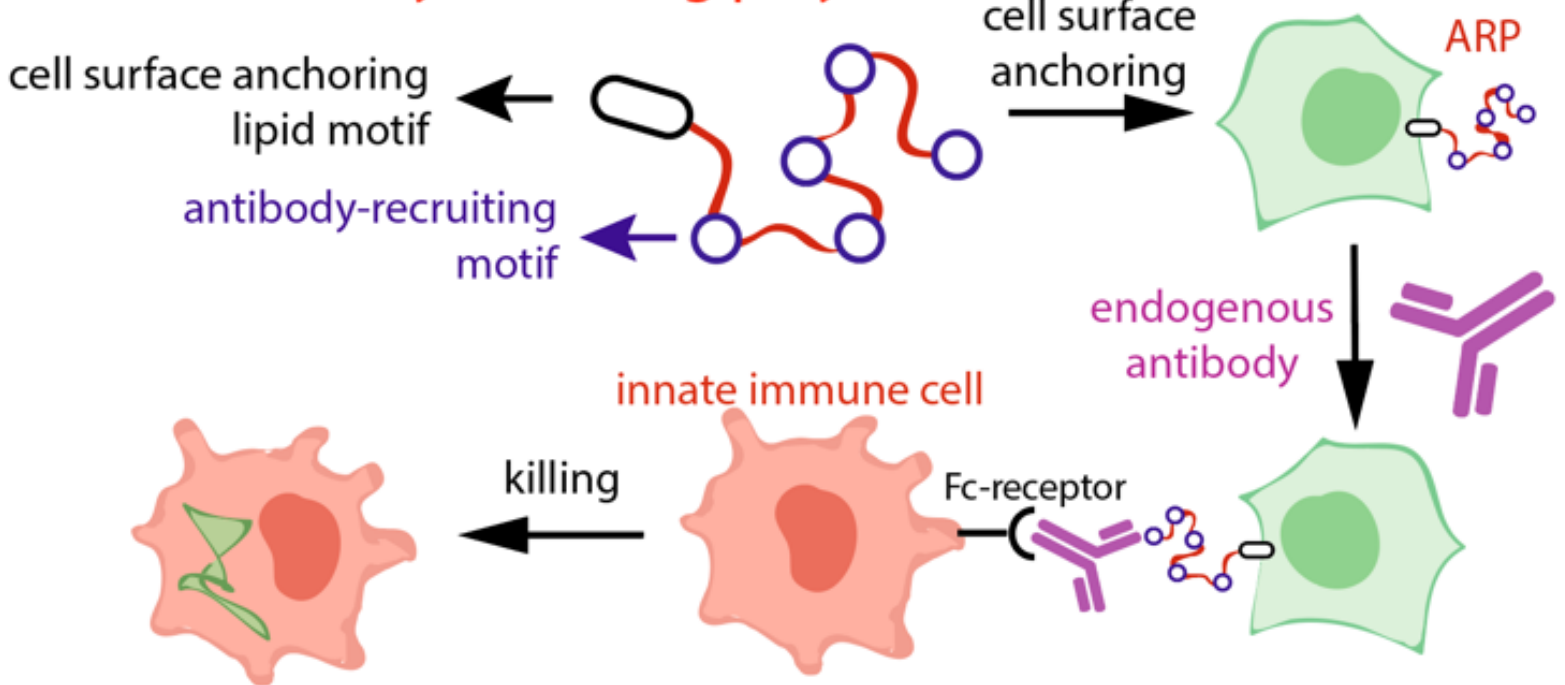

Scheme 1| Antibody-recruiting polymer (ARP) concept. ARPs anchor to the cell surface by hydrophobic insertion of a lipid tail at the ARP chain end into the phospholipid cell membrane. Cell surface anchoring of ARPs triggers recruitment of endogenous antibodies to the cell, thereby flagging the cell for destruction by innate immune effector mechanisms. 


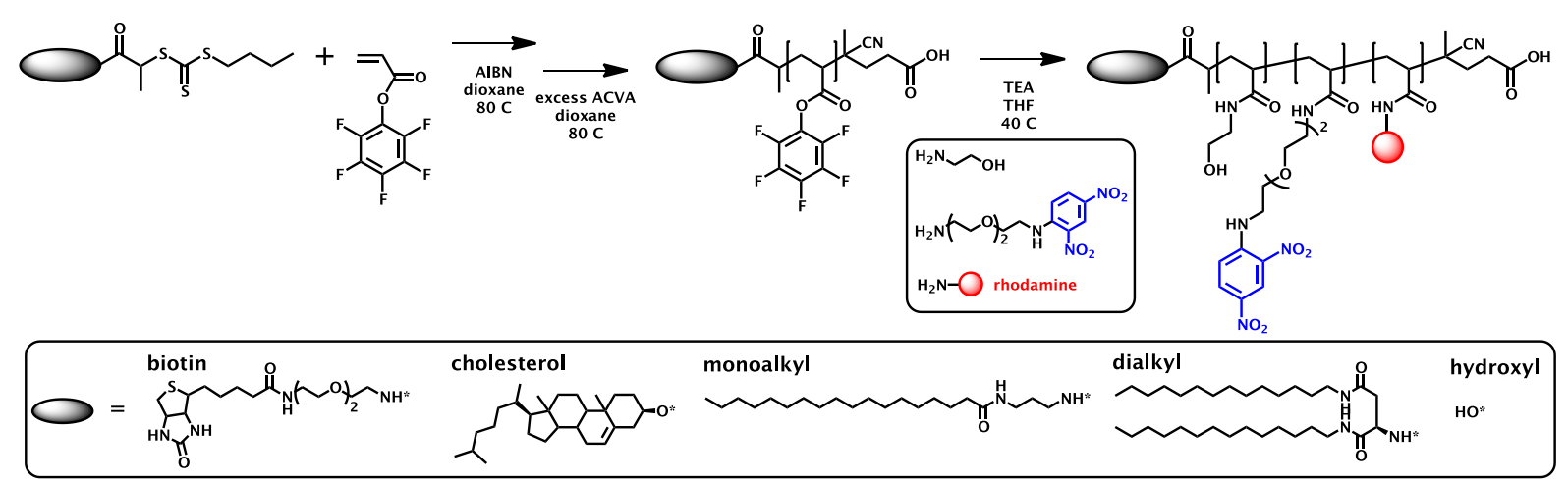

Scheme 2| Antibody-recruiting polymer (ARP) synthesis. A functionalized (i.e. biotin, cholesterol, monoalkylor dialkyl-lipid) chain transfer agent is used for RAFT polymerization of pentafluorophenyl acrylate followed by removal of the trithiocarbonate RAFT end group by treatment with an excess of ACVA. The polymer backbone is subsequently substituted with DNP antibody-recruiting motifs and 2-aminoethanol to render the polymer backbone hydrophilic. For fluorescent labeling rhodamine cadaverine is used. Control polymers are synthesized using a nonfunctionalized chain transfer agent $\left(\mathrm{HO}^{*}\right)$ and/or omitting DNP substitution.

Whereas polymeric constructs that contain multiple hapten copies have been reported earlier, ${ }^{[13-14]}$ it remains elusive whether these are capable to efficiently recruit endogenous antibodies to a cancer cell surface and trigger innate immune killing. Here we focus on ARPs bearing a lipid moiety at one of the chain ends that anchors to cell membranes through hydrophobic interaction. The therapeutic relevance of such ARPs lies within context of intratumoral injection where one aims to engineer the tumor microenvironment to become infiltrated with innate immune cells that induce effector killing. The thus created tumor debris can subsequently serve as a tumor antigen pool which offers opportunities to mount tumor-specific adaptive immunity to fight distal tumors as well as metastases. Of note, intratumoral administration also reduces the risks of unwanted systemic side reaction due to potential immunogenicity of multiple hapten copies onto a polymer backbone.

To start, we focused on how to optimally design ARPs for antibody recruitment. Hereto, pentafluorophenyl acrylate (PFPA) ${ }^{[15]}$ was polymerized by reversible addition-fragmentation chain transfer (RAFT), ${ }^{[16]}$ targeting a final degree of polymerization (DP) of 100 , followed by removal of the trithiocarbonate RAFT end-group by treatment with an excess of 4,4-azobis(4-cyanovaleric acid) (ACVA). For immobilization purpose (vide infra), a biotin-functionalized chain transfer agent (CTA) was used. Hereto, biotin was conjugated to $2,2^{\prime}$-(ethylenedioxy)diethylamine followed by conjugation to 2propanoic acid butyl trithiocarbonate (PABTC). The synthesis route of the polymers is depicted in Scheme 2 and the polymer properties are summarized in Table 1, showing narrow dispersity and thus good control over RAFT polymerization. Experimental characterization data are depicted in the Supporting Information section.

Subsequently, biotin-polyPFPA was substituted with an amine-functionalized dinitrophenol (DNP) derivative (Scheme 2), targeting a degree of substitution (DS; amount of DNP motifs per 100 PFPA repeating units) of respectively 2,5 and 10 . To generate a hydrophilic polymer backbone, all unreacted PFP-esters were converted into hydrophilic repeating units by treatment of the polymer with an excess of 2-aminoethanol. UV-Vis analysis of the resulting polymers after purification revealed that the DNP content was in good agreement with the targeted DS. For comparison, we also synthesized a biotinpolymer without DNP and a monovalent biotin-DNP compound by conjugating biotin to DNP via a diethylene glycol linker (cfr. Supporting Information for the synthesis route). Note that the reason for choosing DNP as antibody-recruiting motif in the context of this work was fostered by the commercial availability of anti-DNP antibodies, which strongly facilitates experimental readout compared to the use of full human serum as a source of endogenous antibodies.

Biolayer interferometry (BLI) was used to investigate the influence of multiple DNP motifs on the avidity of the antibody binding. Hereto, streptavidin-coated sensors were loaded with the monovalent biotinDNP conjugate to test whether the chemistry used for conjugation still allows for antibody binding. When confirmed this was indeed the case, we also used this system to elucidate an optimal concentration 
window for sensor loading and subsequent kinetic analysis (Figure S7) of anti-DNP binding, which was then used to measure the avidity of (polyclonal rabbit) anti-DNP binding to DNP-polymers. Figure 1 shows sensorgrams of anti-DNP binding to DNP-polymer-functionalized sensors. These data demonstrate low non-specific antibody binding to control sensors loaded with control polymers that did not contain DNP. By contrast, DNP-polymers showed strong adsorption and low desorption of anti-DNP. Curve fitting and calculation of the binding avidity $\mathrm{K}_{\mathrm{D}}$ (Table 2), reveals a dramatic increase in binding avidity with increasing DS of DNP on the polymer backbone. Notably, more than a 4-log increase in $K_{D}$ of a DS 10 DNP-polymer compared to monovalent DNP is observed. This can likely be attributed to multivalent binding, ${ }^{[17]}$ as once an antibody dissociates from one DNP motif, it can rapidly bind to a neighboring DNP motif located on the same or adjacent polymer backbone.

Table 1 | Characteristics of the synthesized polymers.

\begin{tabular}{|c|c|c|c|c|c|c|c|}
\hline Polymer & DPtheo & Conv. calc., a [\%] & DPcalc., b & $\mathrm{Mn}^{\text {theo, b }}[\mathrm{Da}]$ & $\mathrm{Mn}^{\mathrm{c}}[\mathrm{Da}]$ & $M w^{c}[D a]$ & $\theta$ \\
\hline biotin-polyPFPA & 110 & 89 & 98 & 23800 & 15200 & 20100 & 1.32 \\
\hline polyPFPA & 100 & 85 & 85 & 20400 & 13200 & 15400 & 1.18 \\
\hline monoalkyl-polyPFPA & 120 & 93 & 112 & 27200 & 12500 & 15900 & 1.27 \\
\hline cholesterol-polyPFPA & 120 & 90 & 108 & 26300 & 16300 & 18100 & 1.11 \\
\hline
\end{tabular}

a: conversion determined by ${ }^{19} \mathrm{~F}-\mathrm{NMR}\left(282 \mathrm{MHz} ; \mathrm{CDCl}_{3}\right)$.

$\mathrm{b}$ : based on conversion and molecular weight of monomer and CTA

c: determined by SEC (THF)

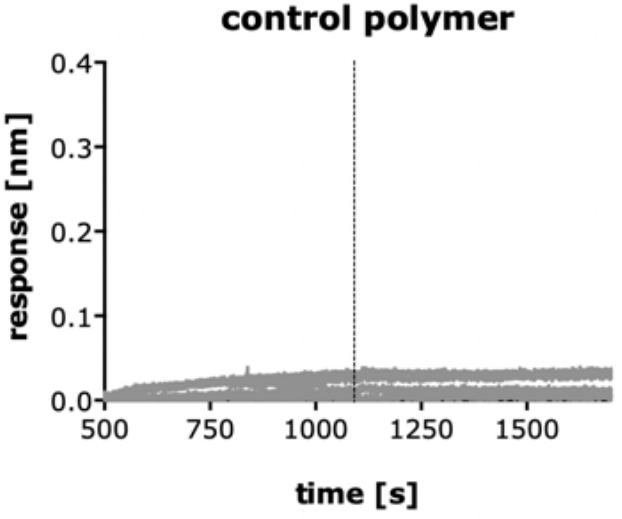

biotin-polyDNP DS5

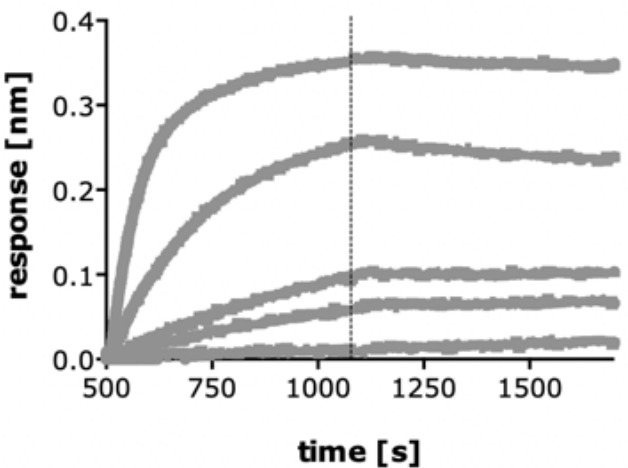

biotin-polyDNP DS2

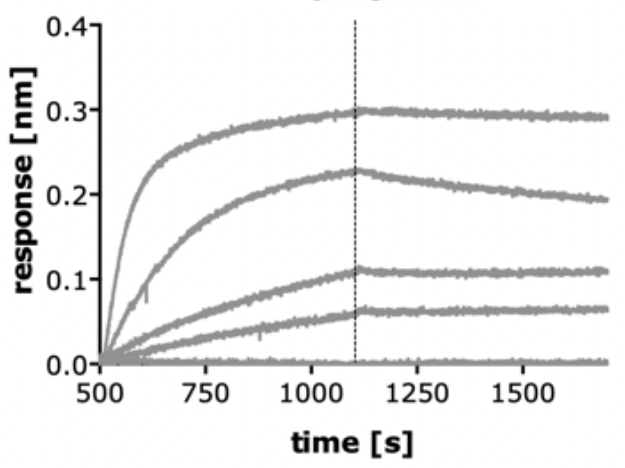

biotin-polyDNP DS10

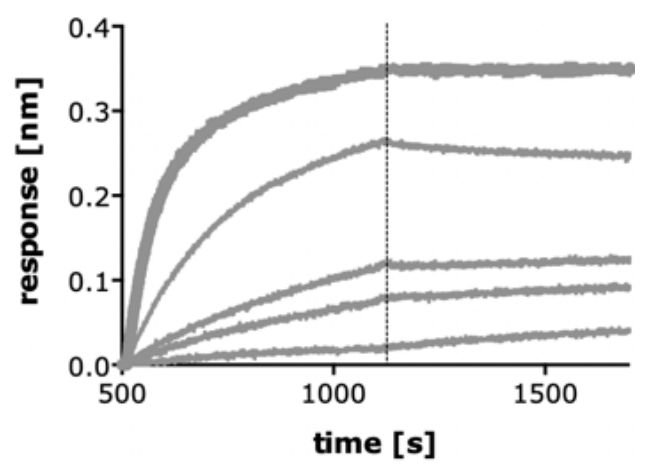

Figure 1 | Multivalency effect on antibody binding to ARPs. Biolayer interferometry sensorgrams of (polyclonal rabbit) anti-DNP binding to streptavidin-coated sensors functionalized with biotinylated polyDNP. A blank biotinylated polymer was used as a control to assess non-specific binding. A dilution series of 22.5, 11.25, 5.6, 2.8 and $0 \mathrm{nM}$ of anti-DNP was measured to calculate $\mathrm{K}_{\mathrm{D}}$ values. Sensors were dipped into the anti-DNP solution at the $500 \mathrm{~s}$ time point, which marks the onset of adsorption. At the $1125 \mathrm{~s}$ time point (dotted line), sensors were dipped into buffer, which marks the onset of desorption. 
Table 2 $\mid$ Calculated $K_{D}$ values and $K_{D}$ error measured by biolayer interferometry (BLI).

\begin{tabular}{lll}
\hline Ligand & $\mathrm{K}_{\mathrm{D}}(\mathrm{M})$ & $\mathrm{K}_{\mathrm{D}}$ error $(\mathrm{M})$ \\
\hline biotin-DNP & $1.12 \cdot 10^{-8}$ & $3.18 \cdot 10^{-11}$ \\
\hline biotin-polyDNP DS2 & $1.23 \cdot 10^{-10}$ & $2.73 \cdot 10^{-12}$ \\
\hline biotin-polyDNP DS5 & $6.80 \cdot 10^{-11}$ & $2.54 \cdot 10^{-12}$ \\
\hline biotin-polyDNP DS10 & $<1.00 \cdot 10^{-12}$ & $<1.00 \cdot 10^{-12}$ \\
\hline
\end{tabular}

These findings prompted us to design ARPs based on a DP 100 polyPFPA backbone substituted with a DS 10 of DNP. As a proof-of-concept, we elaborated on ARPs that can anchor to the cell surface via hydrophobic insertion into the phospholipid cell membrane, inspired by earlier studies that macromolecular amphiphiles containing a lipid motif at their chain end are prone to spontaneous cell membrane anchoring. ${ }^{[18,19]}$ Hereto we synthesized 3 different RAFT CTAs bearing respectively a monoalkyl, dialkyl and cholesterol motif (Scheme 2). In brief, a cholesterol-CTA was synthesized by simple esterification of cholesterol and PABTC. A mono-alkyl-CTA was synthesized by conjugation of stearyl chloride to aminopropanol followed by conjugation to PABTC. A dialkyl-CTA was synthesized by conjugating tetradecylamine to aspartic acid followed by conjugation to PABTC. Note that the choice for a 14 carbon alkyl chain was fostered by earlier reports suggesting that these show improved cell membrane anchoring relative to longer 18 carbon lipid tail based structures. ${ }^{[19]}$ Lipid-CTAs were subsequently used for RAFT polymerization of polyPFPA, followed by DNP modification as detailed above for biotinylated polymers. Note that following trithiocarbonate end-group removal, the polymer was fluorescently labeled with tetramethylrhodamine-cadaverine (DS 1) to allow for detection by fluorescence-based techniques. The resulting polymer properties are summarized in Table 1, while experimental characterization is shown in the Supporting Information section. In all cases well defined narrowly dispersed polymers were obtained, pointing at good control over RAFT polymerization using these lipid-functionalized CTAs.

To investigate the influence of the lipid tail on cell membrane anchoring, CT26 mouse colon cancer cells were pulsed in vitro with the lipid-polymer conjugates for $2 \mathrm{~h}$ at $37^{\circ} \mathrm{C}$, followed by flow cytometry and confocal microscopy analysis. Note that in these experiments, the sample concentration was slightly adjusted based on the fluorescence emission intensity to ensure that the cells received equal doses of fluorescence as the latter parameter was used for quantification purpose. The mean fluorescence intensity (MFI; Figure 2A) values measured by flow cytometry indicated that all lipid-polymer conjugates anchor to cells, unlike the control polymer that lacks a lipid tail. These findings are confirmed by confocal microscopy showing the localization of the fluorescently labeled lipid-polymer conjugates at the cell surface. (Figure 2B) Interestingly, the dialkyl-polymer conjugate shows the highest extent of cellular association. Next, we investigated the ability of the lipid-polymer conjugates to recruit anti-DNP antibodies to the cell surface. Hereto, polymer-treated cells were washed to remove unbound polymer, and subsequently pulsed with AF488-labeled (polyclonal rabbit) anti-DNP followed by flow cytometry (Figure 2C) and confocal microscopy (Figure 2B) analysis. These data unambiguously demonstrate that only conjugates that contain both a lipid tail and DNP motifs are able to recruit anti-DNP to the cell surface. In accordance to the binding of the lipid-polymer conjugate itself, the dialkyl-containing construct is the most proficient in antibody recruitment. Importantly, control polymers that do not contain DNP motifs induce low non-specific binding in the same range as the autofluorescence of the cell. Furthermore, as shown in Figure 2D, a monovalent DNP-dialkyl lipid control conjugate (cfr. Scheme S1 in supporting information for the chemical structure) had very poor anti-DNP recruiting capacity. This could be attributed to the DNP motif being buried too deep into the glycocalyx to allow for the anti-DNP to bind its target epitope, and because of the much lower avidity of anti-DNP towards monovalent DNPconstructs relative to multivalent DNP-constructs as evidenced by biolayer interferometry (vide supra). In analogy with earlier reports by the Bertozzi group, ${ }^{[19]}$ both dialkyl- and cholesterol-based ARP conjugates show efficient cell membrane anchoring, which is translated into efficient antibody 
recruitment. Monoalkyl lipids have been reported to exhibit less efficient cell membrane anchoring, ${ }^{[20]}$ likely due to their lower hydrophobicity and less structural similarity to lipid compounds in the cell membrane. ${ }^{[21]}$ Cholesterol-based conjugates on their hand have been reported to undergo fast exchange from the cell membrane, which might account for the less efficient antibody-recruiting capacity of cholesterol-based ARPs. ${ }^{[22]}$ Using the dialkyl-polyDNP conjugate, we subsequently tested its persistence on the cell surface over prolonged periods of time by treating cells with polymers followed by pulsing with anti-DNP at different time points prior to flow cytometry and confocal microscopy analysis. Note that in these experiments, cells were carefully washed to remove unbound polymer to avoid continuous replenishing of lipid-polymer on the cell surface from lipid-polymer in solution. These experiments (Figure 2E) demonstrate that for at least up to 4 days post dialkyl-polyDNP treatment, antiDNP can bind to the cell surface. Whereas antibody-dependent effector mechanisms occur fairly rapidly (vide infra for ADCP data), we hypothesize that prolonged cell surface display in vivo of ARPs allows for a higher chance of Fc-recognition to occur and innate effector killing to take place. Moreover, our data show that despite the fact that indeed ARPs become endocytosed to a certain extent (as evidenced by the dotted red fluorescent intracellular pattern in the confocal images in Figure 2B), a very significant fraction of ARPs remains on the cell surface and is capable of recruiting anti-DNP to the cell surface for up to 4 days post pulsing of cells with ARPs.
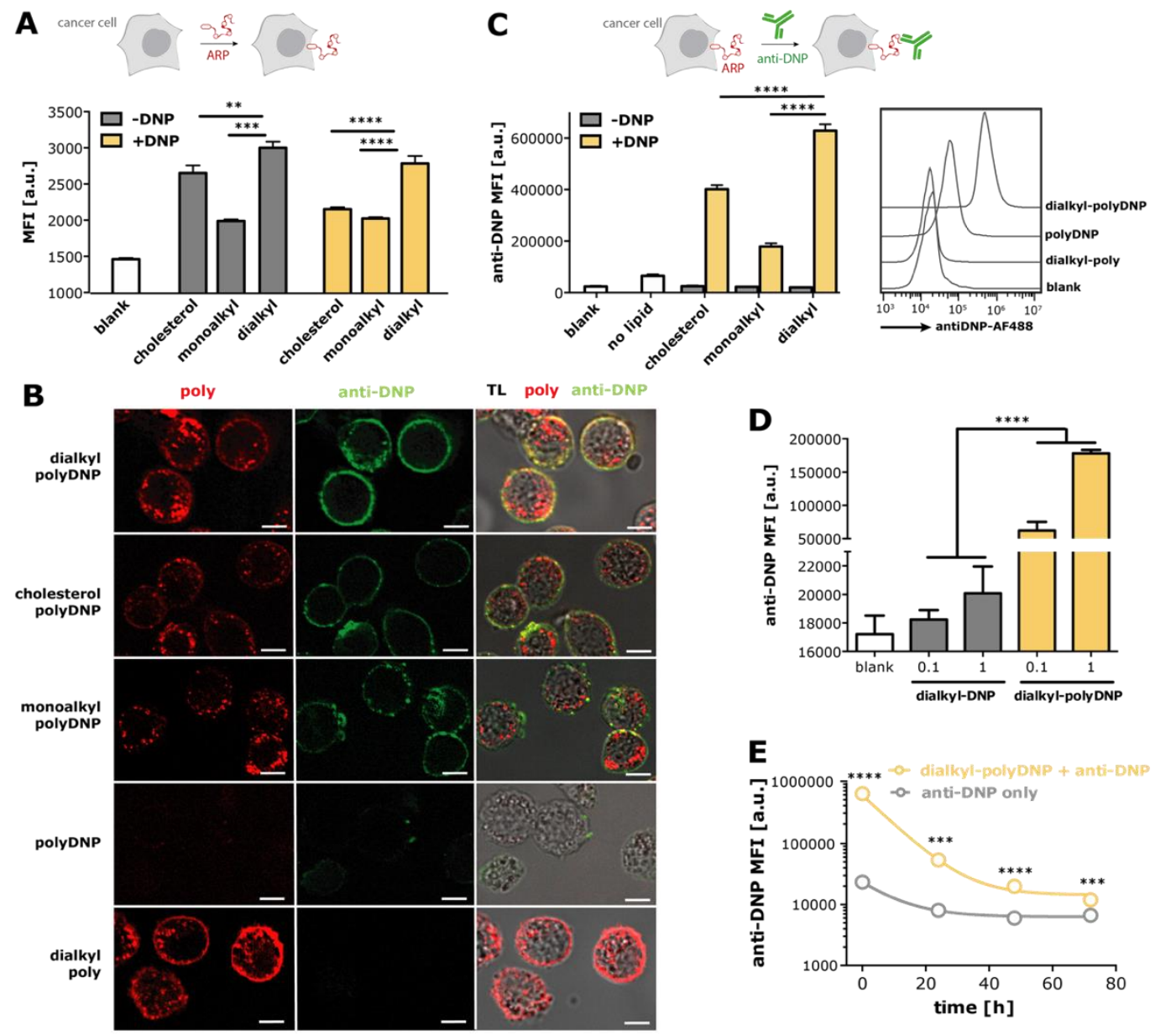

Figure 2 Antibody recruitment to ARP-treated cells. (A) Binding of ARPs through lipid-mediated anchoring to CT26 cells. $\left(n=3 ; 1\right.$ way Anova $\left.{ }^{* * * *}: p<0,0001 ;{ }^{* * *}: p<0,001 ;{ }^{* *}: p<0,0001\right)(B)$ Confocal microscopy images of cells treated with ARPS (red color) and anti-DNP (green color). Scale bar represents $10 \mu \mathrm{m}$. (C) Antibody (antiDNP) recruitment to ARP-treated CT26 cells. Left panel shows a representative flow cytometry histogram of the dialkyl-polyDNP ARP with controls. Right panel compares all ARPs for their ability to recruit anti-DNP antibody to the surface of treated cells. ( $n=3 ; 1$ way Anova $\left.{ }^{* \star \star \star}: p<0,0001 ;{ }^{* \star *}: p<0,001 ;{ }^{* \star}: p<0,0001\right)$ (D) Flow cytometry analysis comparing antibody recruiting capacity between a monovalent dialkyl-DNP conjugate and dialkyl-polyDNP. $\left(n=3 ; 1\right.$ way Anova $\left.{ }^{* * * *}: p<0,0001\right)$ (E) Flow cytometry analysis of cell surface persistence of ARPs. Cells were treated with ARP and pulsed with anti-DNP at different time points. $\left(n=3 ; t\right.$ test $\left.{ }^{\star * \star} p<0,0001 ;{ }^{* * *}: p<0,001\right)$. Note that in all experiments AF488-labeled (polyclonal rabbit) anti-DNP was used. 
Interestingly, earlier reports suggest the cell surface persistence of dialkyl-polymer conjugates to be no more than $24 \mathrm{~h},{ }^{[19,20]}$ whereas at present we demonstrate antibody recruitment for at least 4 days on dialkyl-polyDNP treated cells. However, in these previous works, typically phospholipid-based conjugates where used whereas in our present work we make us of neutral charged amide-linked lipids. These findings highlight the complex influence of lipid-polymer amphiphile structure and membrane anchoring and internalization kinetics and the underlying pathways. Our data indicates that the dialkylpolyDNP conjugate was endocytosed, but at a rate that is sufficiently slow to still allow for antibody recruitment to last over several days. This could be a crucial feature in vivo to allow for prolonged exposure of ARP-treated tumors to antibody recruitment and subsequent innate immune attack. Moreover, our ARP strategy excels in simplicity and dialkyl-polyDNP does not require formulation into higher ordered structures such as fusionogenic liposomes to drive cell surface display. [23]

As a final part of this work, we aimed at demonstrating the ability of ARPs to trigger cancer cell killing by innate effector immune cells. Hereto we performed a functional assay to analyze ARP-mediated phagocytosis (ADCP) by macrophages, triggered by Fc-receptor mediated recognition of clustered antibodies on the surface of an ARP-anchored cancer cell. Hereto we made use of A431 human squamous carcinoma cells and monoclonal human IgG1 anti-DNP to match species and because the IgG1 isotype is the most potent inducer of Fc-mediated effector killing in human. ${ }^{[4]}$ Figure $\mathbf{S 8}$ in Supporting Information confirms that this antibody can also robustly be recruited onto the surface of ARP-treated cells.

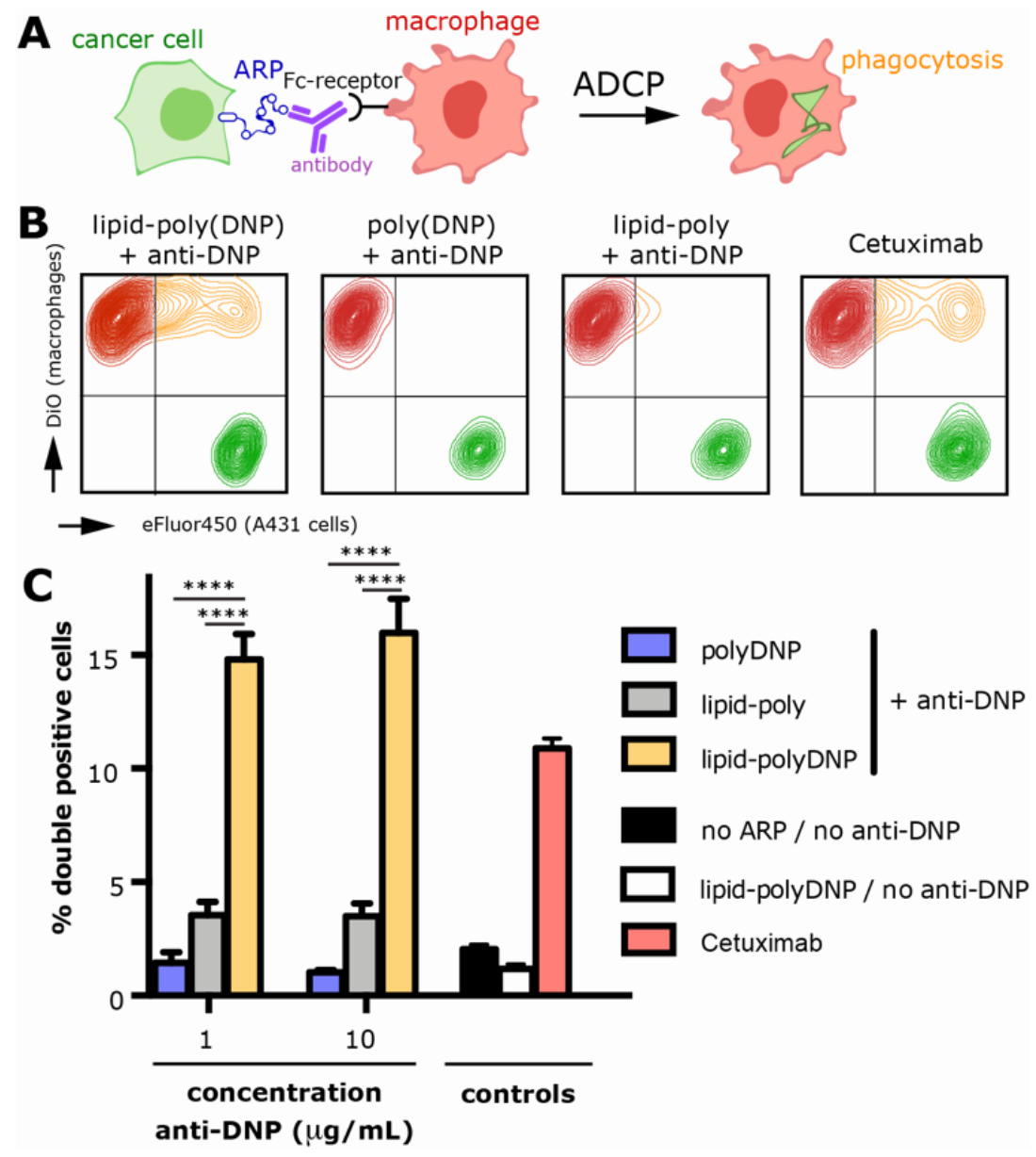

Figure 3 | Innate effector killing of ARP-treated cells (A) Schematic illustration of the ADCP assay. A431 cancer cells are fluorescently labeled with eFluor450 (green color) and pulsed with polymer samples and monoclonal human IgG1 anti-DNP. Macrophages prepared from human peripheral blood monocytes (PBMCs) are fluorescently labeled with $\mathrm{DiO}$ (red color) and co-cultured for $4 \mathrm{~h}$ with the treated cancer cells. (B) Phagocytosis of cancer cells by macrophages provokes a population of double positive cells in the upper right quadrant of flow cytometry scatter plot. (C) Quantification of ADCP efficiency by calculating the percentage of double positive cells in the upper right quadrant in the flow cytometry scatter plots in panel $B(n=3)$. Cetuximab concentration was $10 \mathrm{ng} / \mathrm{mL}$. $(n=3 ; 1$ way Anova $\left.{ }^{* * *}: p<0,0001\right)$ 
To assess ADCP, cells were pulsed with ARPs and anti-DNP, fluorescently labeled with eFluor 450 and co-cultured with macrophages prepared from human peripheral blood mononuclear cells (PBMCs), labeled with DiO. Flow cytometry analysis (Figure 3) clearly shows the presence of a double-positive quadrant, indicating phagocytosis of cancer cells by macrophages, only when cancer cells were treated with dialkyl-polyDNP ARP + anti-DNP. Controls that include lipid-polymer conjugates without DNP or without lipid only induce a low background phagocytosis. Note that the minor background phagocytosis caused by the lipid-poly control could be attributed to a slight extent of toxicity of this amphiphile, thereby triggering macrophage to clear dead/dying cells.

Interestingly, ARPs induce a similar extent of phagocytosis as Cetuximab, a monoclonal antibody that recognizes the epidermal growth factor receptor (EGFR) which is strongly over-expressed by A431 cells and that is known to exert its mechanism of action through Fc-mediated innate effector killing. ${ }^{[4]}$ The ability of ARPs, within a similar experimental context, to be equally potent as a clinically effective drug highlights the potential of ARPs. Moreover, ARPs operate in a receptor-independent way, albeit requiring direct intratumoral injection when envisioning therapeutic translation.

In summary, we have demonstrated the ability of polymer-lipid conjugates containing multiple DNP motifs to highly efficiently anchor to cell membranes and provoke robust recruitment of anti-DNP antibodies to the cell surface in a selective way. The antibody recruitment efficacy of polymeric constructs composed of multiple antibody-recruiting motifs dramatically surpasses control monovalent constructs. The feasibility of this approach was demonstrated to be highly efficient in vitro and proved capable to induce innate effector killing against ARP-treated cells.

\section{Acknowledgements}

BGDG acknowledges the FWO-EOS program for funding. RDC acknowledges Kom Op Tegen Kanker for funding.

\section{References}

[1] A. M. Scott, J. D. Wolchok, L. J. Old, Nat. Rev. Cancer 2012, 12, 278-287.

[2] L. M. Walker, D. R. Burton, Nat. Rev. Immunol. 2018, 18, 297-308.

[3] A. Pincetic, S. Bournazos, D. J. DiLillo, J. Maamary, T. T. Wang, R. Dahan, B.-M. Fiebiger, J. V Ravetch, Nat. Immunol. 2014, 15, 707-716.

[4] N. Gül, L. Babes, K. Siegmund, R. Korthouwer, M. Bögels, R. Braster, G. Vidarsson, T. L. M. ten Hagen, P. Kubes, M. van Egmond, J. Clin. Invest. 2014, 124, 812-823.

[5] W. Wang, A. K. Erbe, J. A. Hank, Z. S. Morris, P. M. Sondel, Front. Immunol. 2015, 6, DOI 10.3389/FIMMU.2015.00368.

[6] C.-H. Lee, G. Romain, W. Yan, M. Watanabe, W. Charab, B. Todorova, J. Lee, K. Triplett, M. Donkor, O. I. Lungu, et al., Nat. Immunol. 2017, 18, 889-898.

[7] P. J. McEnaney, C. G. Parker, A. X. Zhang, D. A. Spiegel, ACS Chem. Biol. 2012, 7, 1139-51.

[8] C. E. Jakobsche, C. G. Parker, R. N. Tao, M. D. Kolesnikova, E. F. Douglass, D. A. Spiegel, ACS Chem. Biol. 2013, 8, 2404-11.

[9] R. T. C. Sheridan, J. Hudon, J. A. Hank, P. M. Sondel, L. L. Kiessling, Chembiochem 2014, 15, 1393-8.

[10] S. Li, B. Yu, J. Wang, Y. Zheng, H. Zhang, M. J. Walker, Z. Yuan, H. Zhu, J. Zhang, P. G. Wang, et al., ACS Chem. Biol. 2018, 13, 1686-1694.

[11] R. P. Murelli, A. X. Zhang, J. Michel, W. L. Jorgensen, D. A. Spiegel, J. Am. Chem. Soc. 2009, 131, 17090-17092.

[12] A. F. Rullo, K. J. Fitzgerald, V. Muthusamy, M. Liu, C. Yuan, M. Huang, M. Kim, A. E. Cho, D. A. Spiegel, Angew. Chemie Int. Ed. 2016, 55, 3642-3646.

[13] S.J. Metallo, R.S. Kane, R.E. Holmlin, G.M. Whitesides, J. Am. Chem. Soc. 2003, 125, 4534-40.

[14] J. Li, S. Zacharek, X. Chen, J. Wang, W. Zhzng, A. Janczuk, P.G. Wang, Bioorg. Med. Chem. 1999, 7, 1549-58. 
[15] A. Das, P. Theato, Chem. Rev. 2016, 116, 1434-95.

[16] C. Boyer, V. Bulmus, T. P. Davis, V. Ladmiral, J. Liu, S. Perrier, Chem. Rev. 2009, 109, 5402-36.

[17] M. Mammen, S. Choi, G. Whitesides, Angew. CHEMIE-INTERNATIONAL Ed. 1998, 37, 27552794.

[18] H. Liu, B. Kwong, D. J. Irvine, Angew. Chemie Int. Ed. 2011, 50, 7052-7055.

[19] E. C. Woods, N. A. Yee, J. Shen, C. R. Bertozzi, Angew. Chem. Int. Ed. Engl. 2015, 54, 15782-8.

[20] K. Kato, C. Itoh, T. Yasukouchi, T. Nagamune, Biotechnol. Prog. 2004, 20, 897-904.

[21] G. van Meer, D. R. Voelker, G. W. Feigenson, Nat. Rev. Mol. Cell Biol. 2008, 9, 112-24.

[22] T. Fritz, M. Voigt, M. Worm, I. Negwer, S. S. Müller, K. Kettenbach, T. L. Ross, F. Roesch, K. Koynov, H. Frey, et al., Chem. - A Eur. J. 2016, 22, 11578-11582.

[23] A. Pulsipher, M. E. Griffin, S. E. Stone, J. M. Brown, L. C. Hsieh-Wilson, J. Am. Chem. Soc. 2014, 136, 6794-6797. 